\title{
Acknowledgement of reviewers 2012
}

C Springer Science+Business Media New York 2013

The Editor-in-Chief, Dr. Linda M. Liau, and Springer would like to acknowledge reviewers' contributions to the journal and offer their sincere thanks.

Saleem I. Abdulrauf

Ty William Abel

Elliot Abemayor

Roger Abounader

Lauren Abrey

John Adler

Nathalie Y. R. Agar

Manish Kumar Aghi

Manmeet Ahluwala

Kenneth Aldape

Brian Michael Alexander

Francis Ali-Osman

Jeffrey C. Allen

Qian An

Mitsuhiro Anan

David W. Andrews

Elena Anghileri

Terri Armstrong

Nils Arvold

Lynn Stuart Ashby

Anthony L. Asher

J. Baehring

Suzanne Baker

Leonora Balaj

Yeshavanth Kumar Banasavadi-

Siddegowda

Igor J. Barani

Fred G. Barker

Garni Barkhoudarian

Jill Barnholtz-Sloan

Valeria Barresi
Ulrich Batzdorf

Luc Bauchet

Oren J. Becher

M. E. Beckner

Christoph P. Beier

Steven Bensinger

Michael E. Berens

A. Tommy Bergenheim

Myriam Bernaudin

Mark Bernstein

Shuguang Bi

Peter McLauren Black

Jashri Blakeley

Deborah T. Blumenthal

Stefan Bluml

Florien W. Boele

John A. Boockvar

Frederick A. Boop

Daniela Annenelie Bota

Eric Bouffet

David Bourne

Dieta Brandsma

Priscilla Brastianos

Daniel J. Brat

William Breitbart

Henry Brem

Steven Brem

Cameron Brennan

William C. Broaddus

Agnieszka Bronisz

Jeffrey N. Bruce
Jack Bui

Peter C. Burger

Nicholas A. Butowski

Thomas Byrne

J. G. Cairncross

Pengxiu Cao

Antoine Carpentier

Jose Carrillo

Rona S. Carroll

Robert Craig Castellino

Maria G. Castro

Robert Cavaliere

Arnab Chakravarti

Marc C. Chamberlain

Jennifer Chan

Michael D. Chan

Steven D. Chang

Samuel Chao

Wassim Chemaitilly

Chu Chen

Clark Chen

Thomas C. Chen

Wei Chen

Shi-Yuan Cheng

Samuel Cheshier

Andrew S. Chi

John Chi

Lawrence S. Chin

Prakash Chinnaiyan

Murali Chintagumpala

E. Antonio Chiocca 
Jin Mo Cho

Yoon-Jae Cho

Chulhee Choi

Arthur P. Chou

Ali K. Choucair

Jennifer L. Clarke

Elizabeth Claus

Timothy F. Cloughesy

Charles S. Cobbs

Bruce H. Cohen

Kenneth Cohen

Aaron A. Cohen-Gadol

Chaim Colen

Brent R. Collett

Luca Colucci-D'Amato

Benjamin Corn

William T. Couldwell

William T. Curry

Johannes Czernin

Lokesh Dalasanur Nagaprashantha

Ricard Damien

Richard Daneman

Sunit Das

Tom Belle Davidson

Faith G. Davis

John F. de Groot

Franco De Monte

Lisa DeAngelis

Waldemar Debinski

Jeffrey D. Demanes

Girish Dhall

Kaijun Di

Curtis A. Dickman

Jorg Dietrich

William P. Dillon

Francesco DiMeco

Oliver Dorigo

Robert Dubrow

Francois Ducray

Eric Duell

Hugues Duffau

Erin M. Dunbar

Ian Dunn

Charles G. Eberhart

Michael J. Eble

Kathleen M. Egan

April F. Eichler

Kamal El Kallab

James Bradley Elder

M. Sam Eljamel

Richard G. Ellenbogen

Benjamin Ellingson

William Elmquist
Marica Eoli

Anat Erdreich-Epstein

Camilo E. Fadul

Xing Fan

Mirco Fanelli

Huaqiang Fang

Haizhong Feng

Ling Feng

Dominique Figarella-Branger

Helen L. Fillmore

Jonathan Lester Finlay

Gaetano Finocchiaro

Paul Graham Fisher

Paul B. Fisher

John Fiveash

Sherry Fox

Allan H. Friedman

Henry S. Friedman

William A. Friedman

Yuejun Fu

Takamitsu Fujimaki

Mitsugu Fujita

Gregory N. Fuller

Daniel W. Fults

Frank B. Furnari

Jaime Gállego-Pérez-Larraya

Sanjiv S. Gambhir

Sharon Gardner

Lars Gerigk

Isabelle M. Germano

Elizabeth Gerstner

Felice Giangaspero

Caterina Giannini

Steven L. Giannotta

Pierre Giglio

Mark R. Gilbert

Mariateresa Giordana

Annarita Giovagnoli

Michael Glantz

Jon Glass

Roberta P. Glick

Joseph C. Glorioso

Catherine Godfraind

Atul H. Goel

Ziya L. Gokaslan

Alexandra J. Golby

Liliana Goumnerova

John Grecula

Grace Griesbach

Sean A. Grimm

Wolfgang Grisold

Christian Grommes

Stuart A. Grossman
Nalin Gupta

Ho-Shin Gwak

Costas G. Hadjipanayis

Walter A. Hall

Kristina Hardy

Griffith Harsh

Peter Hau

Cynthia Hawkins

Monika E. Hegi

Amy Beth Heimberger

Stephanie Heon

Ulrich Herrlinger

Michelle Hickey

Yuichi Hirose

Matthias Holdhoff

Langston Holly

Craig Horbinski

Jethro Hu

Chia-Ho Hua

Erich Huang

Raymond Huang

Shiyong Huang

Martin Kent Hunn

Sara Hurvitz

Dora Ilyasova

Peter Inskip

Keisuke Ishizawa

Mark A. Israel

Fabio Iwamoto

Nada Jabado

Hollie Anne Jackson

Martin Robert Jadus

Rajan Jain

Rakesh Jalali

George I. Jallo

David James

John Anthony Jane

Robert B. Jenkins

Michael D. Jenkinson

Randy L. Jensen

Pengfei Jiang

Mark D. Johnson

Sarah C. Jost

Jeno Julow

Hee-Won Jung

Thomas Kaley

Mitchell R. Kamrava

Ryuichi Kanai

Ekkehard Kasper

Balveen Kaur

Andrew H. Kaye

Daniel F. Kelly

Karl Kelsey 
Santosh Kesari

Mark William Kieran

Turker Kilic

Chae-Yong Kim

Choong Hyun Kim

Chul-Ho Kim

Dong Gyu Kim

Se Hoon Kim

Martin Klein

Bette Kay Kleinschmidt-DeMasters

Arend Koch

Eiji Kohmura

Ricardo Komotar

Douglas Kondziolka

Harley I. Kornblum

David Korones

Christoph Kramm

Teri Nguyen Kreisl

Ali F. Krisht

Carol A. Kruse

Priya U. Kumthekar

Larry E. Kun

Andrew L. Kung

Kaoru Kurisu

Albert Lai

Rose Lai

Karl-Josef Langen

Jean-Philippe Langevin

Joseph L. Lasky

Andrew B. Lassman

John Laterra

Sean Lawler

Edward R. Laws

Eudocia Quant Lee

Ian Yu Lee

John Y. K. Lee

Jong Woo Lee

Jung-Il Lee

Steven Lehrer

Fawn A. Leigh

Jeffrey Leonard

Maciej S. Lesniak

Glenn J. Lesser

Victor A. Levin

Gordon Li

Jian Yi Li

Xiao-Nan Li

Yunqing Li

Linda M. Liau

Terry Lichtor

Keith Ligon

Kevin O. Lillehei

Daniel A. Lim
Michael Lim

Jia-Wei Lin

Michael Linetsky

Mark E. Linskey

N. Scott Litofsky

Arthur K. Liu

Charles Y. Liu

Chong Liu

Tong-Zheng Liu

Xiang Liu

Yang Liu

Zhiyong Liu

Julia Y. Ljubimova

Jay S. Loeffler

Joseph Loftus

Donlin M. Long

Russell R. Lonser

M. Beatriz S. Lopes

Mary Patricia Lovely

Pedro Lowenstein

Mauro Loyo-Varela

Chao Lu

Daniel C. Lu

Yong Lu

Fujun Luo

William Mack

Casey Maguire

Anita Mahajan

Elizabeth Maher

Belinda Mandrell

Peter Manley

Cristina Mantovani

Karen Marcus

Suely Kazue Nagahashi Marie

James M. Markert

Christine Marosi

Warren Mason

Maura Massimino

Gary Mathern

Masao Matsutani

Katherine K. Matthay

Christian Mawrin

David McArthur

William H. McBride

J. Gordon McComb

Rodney D. McComb

Ian E. McCutcheon

Michael W. McDermott

Kerrie Leanne McDonald

Roberta McKean-Cowdin

Roger E. McLendon

Ingo K. Mellinghoff

Thomas E. Merchant
Leonid Metelista

Philippe Metellus

Dominique Michaud

Karine Michaud

Tom Mikkelsen

Ida Milanesi

Siddhartha S. Mitra

Rajendar Mittapalli

Shin Ichi Miyatake

Harsh Mohan

Peter P. Molnar

Maciej Mrugala

Nitin Mukerji

P. Mukherjee

Hermann Muller

Praveen Mummaneni

Karin M. Muraszko

Erin S. Murphy

Louis Burt Nabors

Motoo Nagane

Rajakumar Nagarajan

Goro Nagashima

Seema Nagpal

Mitsutoshi Nakada

Y. Nakazato

Do Hyun Nam

Anil Nanda

Jayant Narang

Atsushi Natsume

Lakshmi Nayak

Edward A. Neuwelt

Phioanh Leia Nghiemphu

Christopher Nimsky

Ryou Nishikawa

Andrew Norden

Alfred T. Ogden

Masasuke Ohno

Hideho Okada

Edward H. Oldfield

Alessandro Olivi

James M. Olson

Jeffrey James Olson

Sara Olson

Antonio M.P. Omuro

Brian Patrick O'Neill

Donald O'Rourke

Andrea Pace

Frank Pajonk

Edward Pan

Ashok Panigrahy

Susan C. Pannullo

Chul-Kee Park 
Deric M. Park

John Park

Ian Parney

Andrew T. Parsa

Chirag Patil

Pritish Pawar

David Peereboom

James Perry

Katherine B. Peters

Claudia Petritsch

Stefan M. Pfister

Joanna Phillips

Peter C. Phillips

Daniel R. Pieper

Russell O. Pieper

Joseph Piepmeier

Scott R. Plotkin

Ian F. Pollack

Mei-Yin Polley

Bruce E. Pollock

Whitney Pope

Kimberly Porter

Nader Pouratian

Kimerly Powell

Michael D. Prados

Robert M. Prins

Martin Andreas Proescholdt

Amy Pruitt

Raj K. Puri

Benjamin Purow

Ibrahim Qaddoumi

Alfredo Quinones-Hinojosa

Alejandra T. Rabadan

Samuel Rabkin

Jeffrey J. Raizer

Zvi Ram

Ganesh Rao

Jasti S. Rao

Renee H. Raynor

Lawrence Recht

Jean Regis

Jacob C. Reijneveld

Zach Reitman

Marc Remke

Sandra A. Rempel

Guillevin Remy

Antoni Ribas

Umberto Ricardi

Jeremy N. Rich

Markus Riemenschneider

Mikael Lee Rinne

David W. Roberts

Giles William Robinson
Fausto J. Rodriguez

Gerald E. Rodts

Lisa Rogers

Federico Roncaroli

Marc K. Rosenblum

Myrna Rosenfeld

Alonzo H. Ross

Tena Rosser

Audrey Rousseau

Martine F. Roussel

James L. Rubenstein

Joshua B. Rubin

J. T. Rutka

Timothy Charles Ryken

Samuel Ryu

Noriko Salamon

Andrea Salmaggi

Ettore Salsano

Maurizio Salvati

Amir Samii

John H. Sampson

Nader Sanai

David I. Sandberg

Jann N. Sarkaria

Hikaru Sasaki

Takashi Sasayama

Raymond Sawaya

Hideyuki Saya

Okay Saydam

Michael Scheurer

David Schiff

David Schiffer

Uwe Schlegel

Rudolph Joseph Schrot

Marc S. Schwartz

Theodore H. Schwartz

Silvia Scoccianti

R. Michael Scott

Laligam N. Sekhar

Michael T. Selch

Nathan R. Selden

Ali Sepahdari

Thomas N. Seyfried

Jason P. Sheehan

Jonas M. Sheehan

Baiyang Sheng

Jonathan Sherman

Paula R. Sherwood

Helen Shih

Masahiro Shin

Nicole Shonka

Dennis Shrieve

Daniel L. Silbergeld
Antonio Silvani

Andrew E. Sloan

Barry Smith

Justin Smith

Zachary A. Smith

Matthew D. Smyth

Riccardo Soffietti

Huijuan Song

Carole Soussain

Robert J. Spinner

Glen H. J. Stevens

Charles D. Stiles

Bryan Strauss

Walter Stummer

Roger Stupp

Lydia Min-Ying Su

Michael E. Sughrue

Justin Sweeney

Walter Taal

Viviane S. Tabar

Ghazaleh Tabatabai

Uri Tabori

Hideo Takeshima

Andrea Talacchi

Taner Tanriverdi

Jiang Tao

Martin Taphoorn

Stephen B. Tatter

Lynne P. Taylor

Michael D. Taylor

Charles Teo

M. Albert Thomas

Reid C. Thompson

Tarik Tihan

Steven A. Toms

Joerg-Christian Tonn

L. Gerard Toussaint

Nhan Tran

Victor K. Tse

Christina Tsien

Cho-Lea Tso

Yong-Kwang Tu

Ugur Ture

Hasan Caglar Ugur

Nicole Ullrich

Martin J. van den Bent

Stefaan Van Gool

Inder Verma

Mariano S. Viapiano

John Villano

Michael A. Vogelbaum

Alfredo Voloschin

Toshihiko Wakabayashi 
Hiroaki Wakimoto

Kyle Walsh

Kevin A. Walter

Beverly C. Walters

Guannan Wang

Yong-gang Wang

Marcus L. Ware

Ronald E. Warnick

Katherine E. Warren

James A. Waschek

Allen Waziri

Jeffrey S. Weinberg

Howard L. Weiner

Stephanie Elizabeth Weiss

Michael Weller

Patrick Y. Wen

Manfred Westphal
Christopher Wheeler

Wolfgang Wick

Joseph Wiemels

John J. Wiencke

Jeffrey Wisoff

Johannes Wolff

Eric T. Wong

Graeme F. Woodworth

Margaret R. Wrensch

Karen Wright

Hui-Yuan Wu

Jing Wu

Julian K. Wu

You Ting Wu

Shuli Xia

Hai Yan

Isaac Yang
Daniel Yanni

Yu Yao

William H. Yong

Daizo Yoshida

Geoffrey Young

W. K. Alfred Yung

Gabriel Zada

Gelareh Zadeh

David Zagzag

Wafik Zaky

Haijun Zhang

Lei Zhang

Rui Zhang

Wei Zhang

Ying Zhang

Yong Zhang

Liang-Fu Zhou 